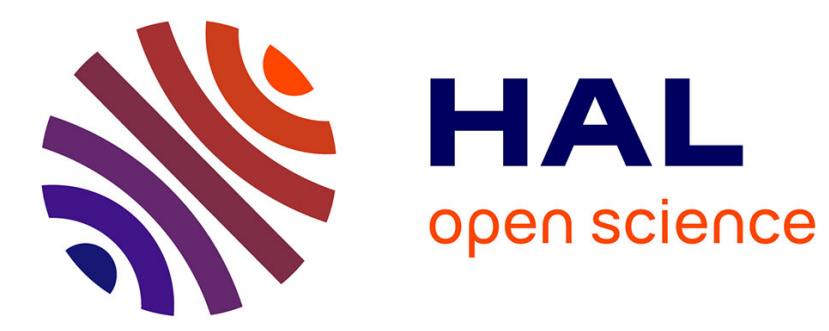

\title{
Image quality assessment based on regions of interest
}

Alireza Alaei, Romain Raveaux, Donatello Conte

\section{To cite this version:}

Alireza Alaei, Romain Raveaux, Donatello Conte. Image quality assessment based on regions of interest. Signal, Image and Video Processing, 2016, 11 (4), pp.673-680. 10.1007/s11760-016-1009-z . hal-01405149

\section{HAL Id: hal-01405149 \\ https://hal.science/hal-01405149}

Submitted on 12 Oct 2018

HAL is a multi-disciplinary open access archive for the deposit and dissemination of scientific research documents, whether they are published or not. The documents may come from teaching and research institutions in France or abroad, or from public or private research centers.
L'archive ouverte pluridisciplinaire HAL, est destinée au dépôt et à la diffusion de documents scientifiques de niveau recherche, publiés ou non, émanant des établissements d'enseignement et de recherche français ou étrangers, des laboratoires publics ou privés. 


\title{
Image Quality Assessment Based on Regions of Interest
}

\author{
A. Alaei · R. Raveaux · D. Conte
}

Received: date / Accepted: date

\begin{abstract}
Most methods in the literature of image quality assessment (IQA) use whole image information for measuring image quality. However, human perception does not always use this criterion to assess the quality of images. Individuals usually provide their opinions by considering only some parts of an image, called regions of interest. Based on this hypothesis, in this research work, a segmentation technique is initially employed to obtain a bi-level image map composed of the foreground and background information. A patch selection strategy is then proposed to choose some particular patches based on the foreground information as the regions of interest for IQA. Three recent IQA methods in the literature are considered to demonstrate the improvement of IQA when using only the extracted regions of interest. To evaluate the impact of the proposed patch selection strategy in various IQA metrics, three publicly available datasets were used for experiments. Experimental results have revealed that our proposal, based on the regions of interest, can improve quality measures of three IQA methods.
\end{abstract}

Keywords Image quality assessment · Image segmentation · Patch selection - Region of interest

\section{Introduction}

Fast development of different technologies has resulted in a massive amount of data generated in the form of digital images in daily life. Smartphone is one among those technologies replacing personal scanners with a

A. Alaei $\cdot$ R. Raveaux $\cdot$ D. Conte

Laboratoire d'Informatique (LI EA6300)

Universit Franois-Rabelais De Tours, France

E-mail: alireza20alaei@gmail.com high growth rate. It is necessary to make the new capture devices more reliable. Therefore, estimating image quality for images captured by such devices is of high demand. As a result, many automatic methods have been proposed in the literature [1,6,9,10,13,16,27-29] to deal with objective image quality assessment (IQA). Based on availability of reference images for computing image quality, the existing methods have been categorized into three main groups called: a) Full Reference (FR), b) No Reference (NR), and c) Reduced Reference $(\mathrm{RR})$ image quality assessment.

Most of the IQA methods presented in the literature use entire image content for estimating image quality [27-29]. However, certain regions of a scene image have superior importance for human observers as well as the human visual system (HVS) for estimating image quality and image degradation $[3,5,6,14,15]$. Based on this hypothesis, many researchers have used different strategies to incorporate various aspects of visual attention for estimating image quality. A number of methods have been developed by integrating saliency awareness models into different objective quality metrics in order to improve image quality prediction performance $[3-5,8$, $9,11,12,15-18,20,21,23-26]$. The approaches in the literature of modelling visual attention can be grouped into two categories: bottom-up and top-down. In the bottom-up approach several models have been introduced to characterize lower level aspects of the HVS employing low-level features, such as colour and local luminance, extracted from scene images to acquire a saliency map $[5,8,16]$. The bottom-up methods for driving visual attention are usually fast, easy and involuntarily controlled $[5,8]$. On the other hand, most of the methods in the literature are based on the topdown approach using higher-level visual abstractions, such as faces, spatial relationships of objects, and seg- 
mentation methods, for automatic saliency detection, weight computation, and region of interest detection in scene images [3-5, 8, 9, 11, 12, 14, 17, 18, 20, 21, 23, 24,26]. In the top-down approach, the value of visual attention has been considered by integrating a computational saliency model to figure out a saliency map for each image. The computed visual saliency map has, further, been integrated into IQA metrics as a weighting map to improve IQA metrics $[5,8,14]$. The accuracy and reliability of the saliency models used to compute the saliency maps are, however, the main concern in saliency-based IQA methods [14,21]. In order to overcome such concerns, some researchers have used an eyetracking system to capture actual visual attention data by freely looking to unimpaired images for boosting the image quality metrics $[5,14,17,20,21,24]$.

In $[5,8,14,17,20,21,24]$, authors have investigated the use of a saliency map obtained by the eye-tracking system in order to provide more reliable image quality assessment models. They have concluded that the use of a saliency map can improve IQA metrics. However, they have further revealed that the improvement is not always significant in some metrics, especially in those that have already provided high performance $[14,17,20$, 21].

In $[3,5,8]$, authors have found that region of interest (ROI) information is useful in improving the performance of image assessment metrics. ROIs are generally driven by the top-down approach using mostly context and semantic information. The most important factors for individuals choosing the ROI were colour, eyes, faces, and camera focus. They have, further, found that human visual attention has a significant relation to the natural scene saliency (NSS) map [8]. In [24], authors have reported that there exists a strong relation between the segmented areas and the saliency map. Also, in [3], authors have experimentally proved that the ROIs have an important effect on improving the IQA metrics. However, their experiments have been conducted based on subjective assessment of data at different stages.

Considering the literature review in this particular domain, it is noted that in most of the methods researchers have attempted to build a separate weighting map as a saliency map, based on local distortions of image content or NSS, to be integrated into the IQA metrics to determine the overall quality score. It is also evident that the application of saliency awareness models can improve image quality prediction performances $[14$, 20]. However, the following facts need to be carefully considered: i) current technologies mostly integrate visual attention into the objective metrics in an ad-hoc manner to increase the performance of IQA metrics [5], ii) most of the existing human visual attention models have specifically been designed to optimize a targeted objective metric in a specific domain and they cannot necessarily be generalized, iii) the reliability of the existing models for image quality assessment has not been completely verified $[15,17,21]$, vi) since computational time is a key factor in real-time applications and massive data processing, the amount of improvement in the metric performance should be balanced with respect to the additional computational costs needed for modeling the visual attention $[6,29], \mathrm{v})$ the amount of research integrating the visual attention and image distortions especially using segmentation based technique is limited $[5,15,17]$, vi) capturing eye-tracking data is expensive and time-consuming and may not fit with all models [5], vii) eye movements of an individual observing a visual scene totally differ based on what task the individual is asked to do or what type of information the individual is looking for in the scene image, viii) artifacts may distract attention away from the natural scene saliency $[17$, $20,21]$, and as such may affect the creation of saliency maps for distorted images by eye-tracking systems.

From the literature review, it is further noted that almost all of the methods have used the entire image along with a visual saliency map, weighting strategy, or a combination of both for estimating image quality. However, despite the integration of a visual saliency map into IQA metrics, meaningful improvement in IQA metrics may not be obtained [17,21]. In this research work, a simple but effective segmentationbased method for detecting/selecting ROIs is proposed. This paradigm comes from the fact that the HVS mostly captures visual/foreground information, neglecting the background area $[3,20,21]$. The concept of perceptual ROI in the literature has been considered as salience or a manually labelled region within a visual scene $[8,5]$.

We, however, consider the bi-level results of an automatic segmentation for defining the ROI. Contrary to the weighting or saliency map extraction methods, which purely depend on the HVS, our proposal is based on statistical analysis of image content. In our method, a foreground/background separation technique is applied to obtain a bi-level image of which the foreground pixels represented in black are called ROIs. There are many sophisticated image segmentation methods in the literature that can provide accurate segmentation results. The Piece-wise Painting Algorithm (PPA) [2] is used to segment scene images into images composed of foreground and background. To demonstrate the significance of using the detected ROIs for IQA, three stateof-the-art (NR and FR) IQA methods are considered for experiments. Furthermore, the mean-shift segmentation [7] as a frequently used segmentation method in 


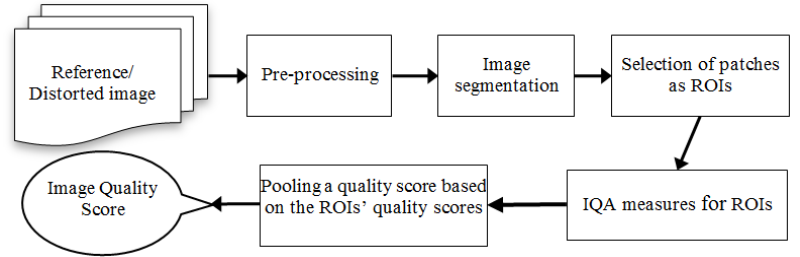

Fig. 1 Block diagram of the proposed system.

the literature is also employed for scene image segmentation to provide a clear observation that the improvement of IQA results does not come from the segmentation but from the patching and patch selection strategy. The results obtained in this research work show the influence of using only ROIs in different IQA methods for predicting image quality. In short, our contributions in this research work are three-fold: (i) we proposed the use of some simple image segmentation techniques to describe the significance of foreground portions of images for IQA, (ii) we proposed a patch selection strategy to choose and incorporate only some particular patches called ROIs for IQA, and (iii) we performed a solid experimental study to signify the impact of our proposed strategy for IQA.

The rest of the paper is laid out as follows: Section 2 describes different elements of the proposed method, especially the part related to foreground extraction and patch selection (ROIs). Section 3 discusses the datasets, experiments, results and comparative analysis. Finally, Section 4 provides some conclusions and future works.

\section{Proposed Method}

\subsection{Image segmentation}

A block diagram of our proposed system is presented in Fig. 1. In our proposed method, the $R G B$ colour images are initially converted into grey-scale ones using a linear combination $(Y=0.21 \cdot R+0.72 \cdot G+0.07 \cdot B)$ of $R G B$ channels, where $R, G, B$ and $Y$ indicate Red, Green, Blue and Grey intensities, respectively. Some pre-processing techniques [28,29], such as mean filtering and down-sampling, are then employed to smooth the reference/distorted images and to decrease the image sizes. The down-sampling is performed by alternately choosing pixels in rows and columns of an image. A colour image and its corresponding grey-scale version are shown in Fig. $2 \mathrm{a}$ and $2 \mathrm{~b}$.

Since in this research work, it is assumed that the foreground information carries more important information compared to the background, a number of patches (ROIs) mainly composed of foreground portions of the input image are selected for assessing the quality of the image. Therefore, in our proposed strategy, following the pre-processing step, a segmentation method is employed to separate foreground from background in the input image. Any image segmentation method in the literature may be used to extract foreground/background information. However, we only need a fast and approximate foreground/background separation technique. Consequently, a state-of-the-art method called Piece-wise Painting Algorithm (PPA) [2] is employed to roughly detect foreground information. To demonstrate the impact of the segmentation method for extracting foreground information in the image quality assessment process, Mean-shift [7] segmentation is also considered for comparison. The results obtained based on both segmentation methods help us to generalize the proposed strategy independently of the segmentation results. The PPA is mainly based on a linear filtering technique and the local Otsu algorithm. The PPA is initially started by dividing the image into a number of vertical stripes of size $s$ from the left to the right direction. Intensity values in each row of a stripe are modified by the average intensity value of that row. Otsu's algorithm is then employed on each stripe separately to binarize the stripes obtained from the input image. As a result, a binary image composed of a number of black and white areas is obtained. The black areas approximately represent foreground information and white portions roughly signify image background [2]. To give an idea of the result of segmentation based on the PPA, a grey-scale scene image and its foreground/background separation result is shown in Fig. $2 \mathrm{~b}$ and 2c, respectively.

Mean-shift holds neighbourhood and minimum pixel gradient magnitude for the segmentation/clustering purpose. More details about the Mean-shift segmentation can be found in [7]. Since, the Mean-shift algorithm may not directly provide two segments, the extracted clusters or regions employing the Mean-shift algorithm are then merged to obtain two segments as foreground and background. The result of the mean-shift segmentation method on the image shown in Fig. $2 \mathrm{~b}$ is depicted in Fig. 2d. It is noted that the result obtained using the Mean-shift segmentation is comparable to the result of the PPA. However, the Mean-shift is a computationally more expensive segmentation method compared to the PPA.

\section{$2.2 \mathrm{ROI} /$ Patch selection}

For patch selection, first, the resultant segmented image is divided into $n$ number of non-overlapping patches of size $p \times p$. A patch $P_{i}$ is selected to contribute to the computation of the quality of input image, if the $P_{i}$ includes a certain amount of foreground (shown in black) 


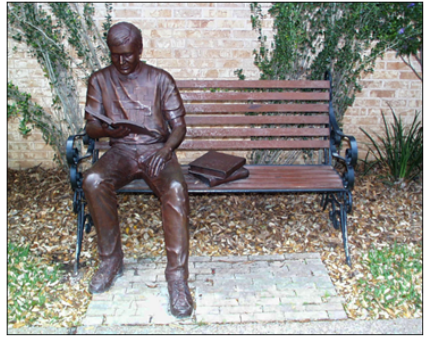

(a)

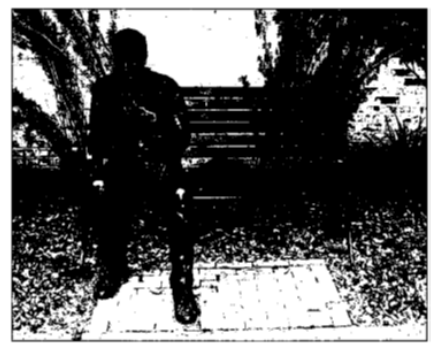

(d)

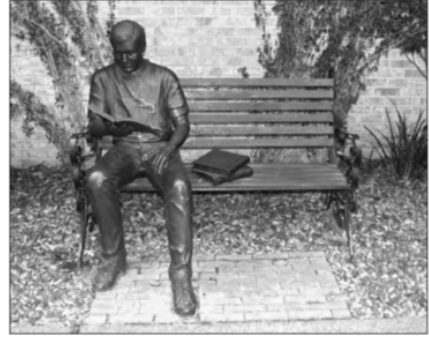

(b)

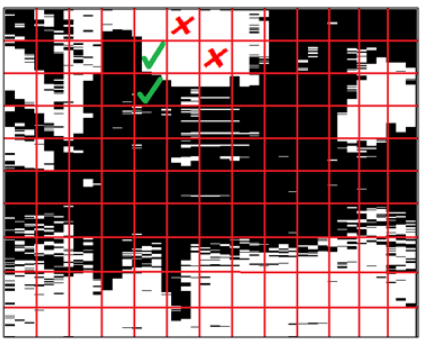

(e)

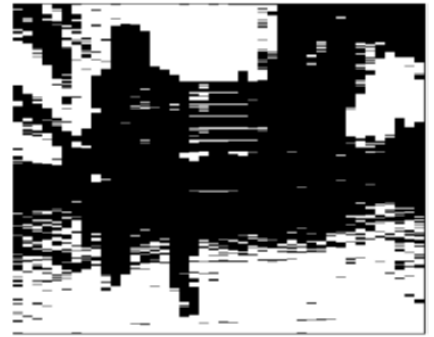

(c)

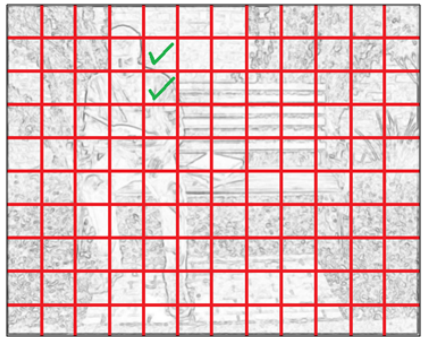

(f)

Fig. 2 a) A sample scene image from the LIVE dataset; b) The grey-scale version of the image shown in (a); c) The segmentation result obtained employing the PPA on (b); d) The segmentation result obtained employing the Mean-shift on (c); e) Two ROIs (selected-patches) are indicated by $\checkmark$, whereas the other two discarded patches are shown by $\boldsymbol{x}$; f) Corresponding two patches of gradient features chosen for computing the image quality are indicated by $\checkmark$.

information. To formulize this process, the following definition is given. Here $\left|F G\left(P_{i}\right)\right|$ means the number of foreground $(F G)$ pixels within the patch $P_{i}$, and $\left|P_{i}\right|$ is the total number of pixels in the patch $P_{i}$.

for $i=1$ to $n \begin{cases}\text { if } T<\frac{\left|F G\left(P_{i}\right)\right|}{\left|P_{i}\right|}<1 & \text { Select } P_{i} \\ \text { otherwise } & \text { Reject } P_{i}\end{cases}$

where $T$ is a threshold, which controls the ratio of the foreground pixels in a patch to the size of the patch. Employing the patch selection strategy with $T=0.25$ on the four patches shown in Fig. 2e, two ROIs/selectedpatches and two discarded patches are indicated by different marks. To demonstrate the impact of patch section on features, the gradient magnitude features of the image shown in Fig. 2b are depicted in Fig. 2f. From Fig. 2f, it is revealed that the important information concerning the gradient magnitude features can be obtained from the foreground and the transition between foreground and background and vice versa; our proposed patch selection strategy is able to pick up this information to use for IQA.

\subsection{ROI image quality measures}

ROI-based IQA can be applied on the top of the FR and NR IQA methods. In this research work, the FSIM [29], GMSD [28] and QAC [27] are considered as the baseline for IQA. Feature similarity (FSIM) [29] and gra- dient magnitude similarity deviation (GMSD) [28] are two state-of-the-art FR IQA methods with promising results on the publicly available datasets. In the FSIM, phase congruency (PC) and gradient magnitude (GM) features have been used for predicting image quality. An average weighting strategy based on PC has been employed to obtain the final quality score of a whole image [29]. The gradient magnitude (GM) feature alone has also been considered for assessing image quality in the GMSD [28]. In the GMSD method, standard deviation statistics have been employed to compute the quality of a whole image. In our proposed modification of FSIM [29] and GMSD [28], respectively called MFSIM and MGMSD, the PC and GM features are extracted from each selected patch in order to estimate patch quality.

Quality-aware clustering (QAC) method is a sophisticated state-of-the-art method proposed for NR IQA in [27]. K-means clustering has been employed to create a knowledge-base representing image quality at different levels. Difference of Gaussian (DoG) feature has been used for patch characterization. Similarities between the extracted DoG features and the cluster centres in the knowledge-base model have been considered to determine patch quality. An average weighting pooling strategy has been used to obtain the final image quality [27]. In the proposed modification of the IQA (MQAC), similar to the IQA, DoG features are computed to estimate the quality of selected patches (ROIs). 


\subsection{Pooling strategy}

In the FR and NR IQA methods, different pooling strategies, such as average pooling, weighted pooling, and standard deviation pooling, have been performed to obtain a quality score for an image from a local quality map [29,28], or from a set of patches, whose qualities are known [27]. In this research work, a conventional average pooling strategy is used to obtain a quality score for an image from the quality values obtained for the selected-patches/ROIs that explore the local qualities of an image. The quality values for the ROIs are obtained based on the corresponding pooling strategies used in the FR/NR IQA methods considered for the experiment in this research work. Therefore, for each selected patch $P_{i}$ extracted from the bi-level image, the corresponding patch in the original image is considered. A feature extraction technique, such as PC, GM and DoG, is then employed to characterize the ROIs. If a FR IQA is used for measuring the quality of the patch $P_{i}$, a local quality map called $L Q M P_{i}$ is computed. A pooling strategy, such as standard deviation [28], or average pooling [29], is employed to obtain a patch quality score $\left(P Q S_{i}\right)$ for the patch $P_{i}: P Q S_{i}=\operatorname{Pooling}\left(L Q M P_{i}\right)$.

As already mentioned, the first pooling is employed at the patch level to obtain a quality score for each selected-patch. In a NR IQA, for example in the QAC method [27], the feature vector extracted from a ROI is used to obtain the patch quality score based on the QAC [27]. To obtain the final image quality score $(I Q S)$ for an image, an average pooling is then employed on all the quality scores $\left(P Q S_{i}\right)$ obtained for the pateches (ROIs).

\section{Experiment Results and Discussion}

\subsection{Datasets and metrics of evaluation}

To evaluate the performance of the proposed strategy, LIVE [22], TID2008 [19], and IBBI [15], as three widely used datasets in the literature for IQA, were considered for experiments. Experimentation on these datasets provides a fair and direct comparison to the state-of-theart results. The IBBI dataset further contains visual saliency maps provided by the eye tracking mechanism. Experimentation on this dataset can provide a comparison to the use of the visual saliency map as the weighting map, integrated with the IQA metrics.

In most of the research works in the literature, the Pearson linear Correlation Coefficient (PCC), Spearman Rank order Correlation coefficient (SRC), and Root Mean Square Error (RMSE) have widely been used for evaluation of different IQA methods. Better performance is indexed by the values close to 1 for the PCC and SRC, whereas the value of RMSE should be close to 0 .

\subsection{Implementation and parameters setting}

The original FSIM [29], GMSD [28], and QAC [27] (without employing the segmentation and patch selection), and their respective modified version integrating the segmentation and patch section strategy (MFSIM, MGMSD, and MQAC) were considered for experiments. As there are three parameters in the proposed strategy, an extensive range of experiments was performed to study the impact of each parameter on the results obtained from our proposed method. These parameters are the width of stripes $(s)$, the size of patches $(p \times p)$, and the threshold $T$. While employing the PPA, the width of stripes $(s)$ should be small enough to take care of intensity variation in an image. In our experiments, we considered different values, such as $2.5 \%$ and $5 \%$, for the width of stripes $(s)$ and obtained the results. We noted that there was no significant change in the results obtained when $s$ was set as $2.5 \%$ or $5 \%$ of the image width in our experiments. Therefore, in the implementation of the proposed method, $s$ was set to $2.5 \%$ of the image width. To give an idea of the impact of patch size $(p \times p)$ in the proposed patch selection strategy, a wide range of values was considered for the patch size to compute results. Also, different values were considered for the parameter $T$ to analyse the significance of foreground information for estimating image quality in different methods. The results are discussed in the next subsection in detail.

\subsection{Results and discussion}

In this research work, a number of experiments were initially conducted to analyze the impact of foreground information, considering different values of parameter $T$ in our proposed method. The PPA was used for the foreground extraction. The evaluation metrics were computed in the form of PCC and the experimental results are demonstrated in Fig. 3, 4 and 5. The results obtained from the original QAC [27], GMSD [28], and FSIM [29] without employing the segmentation and patch selection are also reported. From the results shown in Fig. 3, we noted that the proposed MQAC outperforms the original QAC metric [27] considering all three datasets used for the experiments when different values of less than 0.7 were considered for $T$. The best results were obtained when $T$ was set to 0 for the LIVE and 


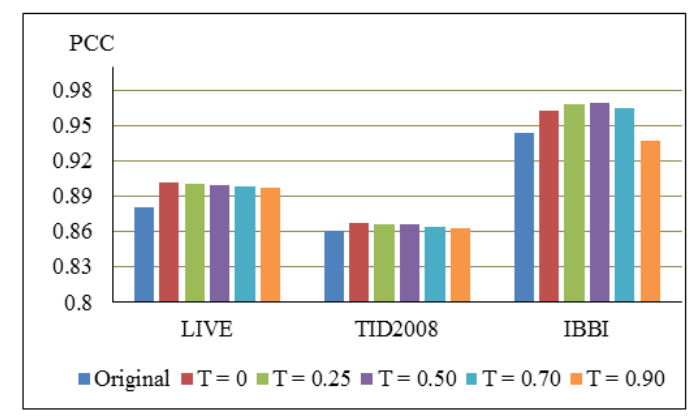

Fig. 3 The results obtained employing the MQAC on different datasets, considering different values of $T$ and patches of size $8 \times 8$ when the PPA is applied for segmentation.

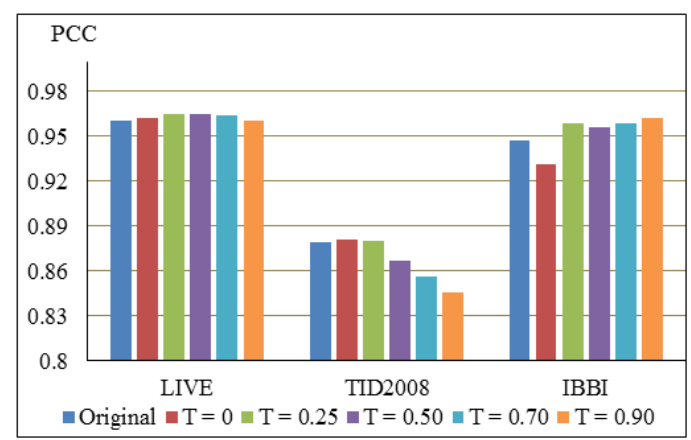

Fig. 4 The results obtained employing the MGMSD on different datasets, considering different values of $T$ and the patches of size $60 \times 60$.

TID2008 datasets, whereas in the IBBI dataset the best performance was achieved when $T$ was set to 0.50 . The results obtained based on the MGMSD considering different values of parameter $T$, are shown in Fig. 4. Fig. 4 demonstrates that the MGMSD provided the best performance on all the three datasets compared to the GMSD [28], when $T$ was considered as 0.25 . From the results shown in Fig. 5 , it can be noted that by employing the MFSIM on the LIVE dataset the best results were obtained when $T=0.25$.

The impact of different patch sizes on the results of the MGMSD method on the LIVE dataset was also analysed and the results are shown in Fig. 6. From Fig. 6, we noted that the best results were obtained using $60 \times 60$ for the patch size. However, other patch sizes also provided very close results compared to the coventional GMSD method.

From the results obtained on the LIVE dataset, it is evident that the proposed MQAC, MGMSD, and MFSIM provide better performance compared to the QAC, GMSD, and FSIM. The improvements were obtained by using mainly the foreground information in the form of ROIs for IQA. The improvement of the results on the TID2008 datasets is less significant compared to the results on the LIVE and IBBI datasets.

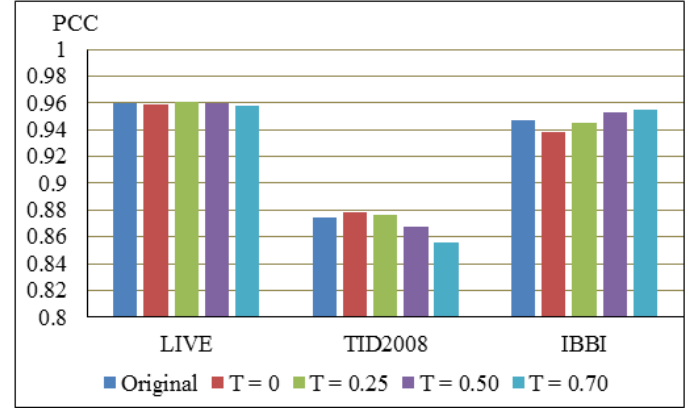

Fig. 5 The results obtained employing the MFSIM on different datasets, considering different values of $T$ and the patches of size $60 \times 60$.

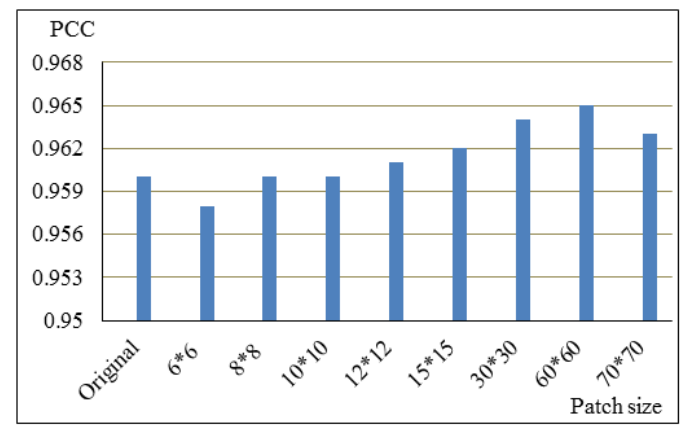

Fig. 6 The results obtained employing the MGMSD on the LIVE dataset, considering different patch-sizes when $T=$ 0.50 .

We further performed experiments using the meanshift, instead of the PPA, for segmentation. The results obtained employing the mean-shift for foreground extraction were comparable with the results achieved using the PPA. However, the time processing cost of the mean-shift was considerably higher than the PPA.

\subsection{Comparative analysis}

The comparison of the results obtained from the proposed MQAC, MGMSD, and MFSIM as well as the QAC, GMSD, and FSIM are demonstrated in Table 1. From Table 1, it is evident that the proposed MQAC method improved the SRC metric from 0.879 to 0.901 on the LIVE dataset. This means an improvement of $2.2 \%$ for the SRC metric was achieved when the proposed patch selection strategy was employed. The improvement is $1.7 \%$ and $1.1 \%$ on the IBBI and TID2008 datasets, respectively.

Furthermore, the results obtained from the proposed MGMSD and MFSIM methods on the IBBI dataset are compared to the results obtained from the GMSD and FSIM by integrating the visual saliency maps (saliency map GMSD and FSIM) in Table 1. The results presented in Table 1 clearly demonstrate that integrating 
Table 1 Comparison of the results obtained employing the MGMSD, MFSIM and MQAC, their corresponding original ones, and integrating the visual saliency maps of the images in the IBBI dataset to the GMSD and FSIM (NA = Not Available).

\begin{tabular}{|c|c|c|c|c|c|c|c|c|c|}
\hline Dataset & $\begin{array}{l}\text { Metric / } \\
\text { Method }\end{array}$ & $\begin{array}{c}\text { original } \\
\text { GMSD }\end{array}$ & $\begin{array}{c}\text { saliency map } \\
\text { GMSD }\end{array}$ & $\begin{array}{l}\text { proposal } \\
\text { MGMSD }\end{array}$ & $\begin{array}{c}\text { original } \\
\text { FSIM }\end{array}$ & $\begin{array}{c}\text { saliency map } \\
\text { FSIM }\end{array}$ & $\begin{array}{l}\text { proposal } \\
\text { MFSIM }\end{array}$ & $\begin{array}{c}\text { original } \\
\text { QAC }\end{array}$ & $\begin{array}{c}\text { proposal } \\
\text { MQAC }\end{array}$ \\
\hline \multirow{3}{*}{ LIVE } & RMSE & 7.62 & NA & 7.15 & 7.68 & $\mathrm{NA}$ & 7.55 & 12.80 & 11.69 \\
\hline & $\mathrm{PCC}$ & 0.960 & NA & 0.965 & 0.960 & NA & 0.961 & 0.881 & 0.902 \\
\hline & $\mathrm{SRC}$ & 0.960 & NA & 0.965 & 0.963 & NA & 0.964 & 0.879 & 0.901 \\
\hline \multirow{3}{*}{ TID2008 } & RMSE & 0.640 & $\mathrm{NA}$ & 0.634 & 0.653 & $\mathrm{NA}$ & 0.648 & 0.804 & 0.790 \\
\hline & PCC & 0.879 & NA & 0.881 & 0.874 & NA & 0.876 & 0.861 & 0.867 \\
\hline & $\mathrm{SRC}$ & 0.891 & NA & 0.893 & 0.880 & NA & 0.882 & 0.838 & 0.849 \\
\hline \multirow{3}{*}{ IBBI } & RMSE & 7.38 & 6.83 & 6.33 & 7.42 & 6.85 & 6.82 & 8.27 & 6.14 \\
\hline & $\mathrm{PCC}$ & 0.947 & 0.955 & 0.962 & 0.947 & 0.955 & 0.955 & 0.944 & 0.969 \\
\hline & $\mathrm{SRC}$ & 0.939 & 0.949 & 0.958 & 0.938 & 0.949 & 0.952 & 0.937 & 0.954 \\
\hline
\end{tabular}

Table 2 Average computation times obtained employing different methods on each image of the LIVE dataset.

\begin{tabular}{cc}
\hline Method & Execution time $(\mathbf{m s})$ \\
\hline FSIM & 487 \\
Proposed MFSIM & 531 \\
GMSD & 50 \\
Proposed MGMSD & 89 \\
QAC & 1571 \\
Proposed MQAC & 1211 \\
\hline
\end{tabular}

visual saliency maps with the GMSD [28] and FSIM [29] considerably has improved the objective quality metrics compared to the original results. However, the results obtained employing our proposed MGMSD and MFSIM methods have provided the best performance.

From the results obtained from our proposed strategy based on the use of ROIs for IQA, we can conclude that in all the cases the use of ROIs for IQA has improved the IQA metrics. The improvement in the IQA metrics, which have already provided higher results, is less significant. This exactly in-line with the finding reported in the literature when eye-tracking data and complex visual attention models were used [14]. Furthermore, it can be mentioned that integrating simple segmentation followed by patch selection strategy with IQA methods provided quite comparative IQA results compared to the use of eye-tracking data as visual saliency maps for estimating objective image quality.

\subsection{Time complexity analysis}

In real-time applications of IQA the complexity of the systems is an important factor. From the literature it can be noted that visual attention models can improve IQA; however, the time complexity of computing visual attention models is always of major concern.

To provide an idea of the time complexity of our proposed model, its time complexity was further computed. Theoretically, if we consider that an image contains $N$ pixels, the PPA has a complexity of $O(N)$, as only a thresholding procedure would be employed on the input image. However, the time complexity of the mean-shift is $O\left(M N^{2}\right)$, where $M$ is the number of iterations. As integration of the PPA and patch selection techniques into the IQA methods does not affect their time complexities in terms of including a higher order of complexity, the PPA was used in our proposed method for segmentation. To further compute the execution time, MATLAB implementation of all the IQA methods was run on a Desktop PC with 16GB RAM and Intel Core 2 DUO CPU@3GHz. The average execution times on each image of the LIVE dataset for each IQA method are shown in Table 2. From Table 2, it is noted that the proposed MQAC ran $360 m s$ faster than the QAC [27]. This is because in the QAC all the patches were considered to estimate image quality, whereas in the MQAC a subset of all the patches as ROIs were considered for IQA. The computation time for the other methods is also comparable. The difference is only the time needed to perform the segmentation.

\section{Conclusions}

In this research work, a new patch selection strategy based on foreground information is proposed for IQA. The impact of patch selection basaed on foreground information for estimating image quality in scene images was studied. Experiments on different datasets provided impressive results showing the practical use of the proposed strategy. The reliability of the assumption that using only ROIs can provide accurate image quality was also demonstrated. We noted that our proposal using the ROIs for predicting image quality achieves better results compared to the use of a whole image for IQA. Concerning the large size images, employing the proposed strategy can drastically reduce the number of patches, which consequently helps to obtain IQA scores faster. It is worth noting that on an average, only $25 \%$ of the patches were considered in the MQAC, providing faster and more accurate results compared to the QAC. 


\section{References}

1. A.A. Abdelouahad, M.E. Hassouni, H. Cherifi, and D. Aboutajdine. Reduced reference image quality assessment based on statistics in empirical mode decomposition domain. Signal, Image and Video Processing, 8(8):16631680, 2014.

2. A. Alaei, U. Pal, and P. Nagabhushan. A new scheme for unconstrained handwritten text-line segmentation. Pattern Recognition, 44(4):917-928, 2011.

3. H. Alers, J. Redi, H. Liu, and I. Heynderickx. Studying the effect of optimizing image quality in salient regions at the expense of background content. Journal of Electronic Imaging, 22(4), 2013

4. A. Beghdadia, M.-C. Larabib, A. Bouzerdoumc, and K.M. Iftekharuddind. A survey of perceptual image processing methods. Signal Processing: Image Communication, 28:811-831, 2013.

5. P. Le Callet and E. Niebur. Visual attention and applications in multimedia technologies. Proceedings of the IEEE, 101(9):2058-2067, 2013.

6. D. M. Chandler. Seven challenges in image quality assessment: Past, present, and future research. ISRN Signal Processing, 2013:53pp, 2013.

7. D. Comaniciu and P. Meer. Mean shift: A robust approach toward feature space analysis. IEEE Trans. on Pattern Analysis and Machine Intelligence, 24(5):603-619, 2002.

8. U. Engelk and P. Le Callet. Perceived interest and overt visual attention in natural images. In Signal Processing: Image Communication, 2015.

9. B. Ghanem, E. Resendiz, and N. Ahuja. Segmentationbased perceptual image quality assessment (spiqa). In Proceedings of the International Conference on Image Processing, pages 393-396, 2008.

10. K. Gu, S. Wang, G. Zhai, S. Ma, X. Yang, and W. Zhang. Content-weighted mean-squared error for quality assessment of compressed images. Signal, Image and Video Processing, 10(5):803-810, 2016.

11. A. Guo, D. Zhao, S. Liu, X. Fan, and W. Gao. Visual attention based image quality assessment. In Proceedings of the 18th IEEE International Conference on Image Processing, pages 3297-3300, 2011.

12. L. Itti and C. Koch. Computational modelling of visual attention. Nature Reviews Neuroscience, 2(3):194203, 2001.

13. J. Li, L. Zou, J. Yan, D. Deng, T. Qu, and G. Xie. Noreference image quality assessment using prewitt magnitude based on convolutional neural networks. Signal, Image and Video Processing, 10(4):609-616, 2016

14. H. Liu, U. Engelke, W. Junle, P. Le Callet, and I. Heynderickx. How does image content affect the added value of visual attention in objective image quality assessment? IEEE Signal Processing Letters, 20(4):355-358, 2013.

15. H. Liu, J. Wang, J. Redi, P. Le Callet, and I. Heynderickx. An efficient no-reference metric for perceived blur. In Proceedings of the 3rd European Workshop on Visual Information Processing (EUVIP), pages 174-179, 2011.

16. A. Mittal, A.K. Moorthy, A.C. Bovik, and L.K. Cormack. Automatic prediction of saliency on jpeg distorted images. In International Workshop on Quality of Multimedia Experience, pages 195-200, 2011.

17. A. Ninassi, O. Le Meur, P. Le Callet, and D. Barbba. Does where you gaze on an image affect your perception of quality? applying visual attention to image quality metric. In Proceedings of the 15th IEEE Int. Conf. on Image Processing (ICIP), pages 169-172, 2007.
18. W. Osberger, N. Bergmann, and A. Maeder. Automatic image quality assessment technique incorporating higher level perceptual factors. In Proceedings of the Int. Conf. on Image Processing, volume 3, pages 414-418, 1998.

19. N. Ponomarenko, V. Lukin, A. Zelensky, K. Egiazarian, M. Carli, and F. Battisti. Tid2008 - a database for evaluation of full-reference visual quality assessment metrics. Advances of Modern Radioelectronics, 10:30-45, 2009.

20. J. Redi, H. Liu, P. Gastaldo, R. Zunino, and I. Heynderickx. How to apply spatial saliency into objective metrics for jpeg compressed images? In Proceedings of the 16th IEEE International Conference on Image Processing (ICIP), pages 961-964, 2009.

21. J. Redi, H. Liu, R. Zunino, and I. Heynderickx. Interactions of visual attention and quality perception. In ISEST/SPIE Electronic Imaging, 2011.

22. H.R. Sheikh, Z. Wang, L. Cormack, and A.C. Bovik. Live image quality assessment database release 2, 2006.

23. Y. Tong, H. Konik, F.A. Cheikh, and A. Tremeau. Full reference image quality assessment based on saliency map analysis. Journal of Imaging Science and Technology, 54(3):305031-305034, 2010.

24. J. Wang, M. Barkowsky, and V. Ricordeland P. Le Callet. Quantifying how the combination of blur and disparity affects the perceived depth. Human Vision and Electronic Imaging, 2011.

25. J. Wang, D.M. Chandler, and P. Le Callet. Quantifying the relationship between visual salience and visual importance. In Proceedings of SPIE Human Vision and Electronic Imaging, volume 7527, 2010.

26. Z. Wang and A.C. Bovik. Embedded foveation image coding. IEEE Transactions on Image Processing, 10(10):13971410, 2001.

27. W. Xue, L. Zhang, and X. Mou. Learning without human scores for blind image quality assessment. In IEEE Conference Computer Vision Pattern Recognition (CVPR), pages 995 - 1002, 2013.

28. W. Xue, L. Zhang, X. Mou, and A.C. Bovik. Gradient magnitude similarity deviation: A highly efficient perceptual image quality index. IEEE Transactions on Image Processing, 23(2):684-695, 2014.

29. L. Zhang, L. Zhang, X. Mou, and D. Zhang. Fsim: A feature similarity index for image quality assessment. IEEE Transactions on Image Processing, 20(8):2378-2386, 2011. 